\title{
Year in Review 2014: Asthma
}

\author{
Kathleen M Deakins MHA RRT-NPS FAARC
}

\author{
Introduction \\ Programs and Guidelines \\ Diagnosis and Treatment \\ Diagnosis \\ Treatment \\ Novel Therapies \\ Management \\ Disease Management Programs \\ Environmental Remediation in Asthma Management \\ Summary
}

\begin{abstract}
Asthma continues to be recognized as a well-known respiratory disease requiring complex management. Asthma is assessed and treated by clinicians across the continuum. The interest in evidence-based recommendations for diagnosis, treatment, and long-term management is ongoing and essential for aligning clinical practice with its changes. The purpose of this review is to provide updates from recent literature on asthma for clinicians. Key words: asthma self-management; asthma phenotypes; bronchial thermoplasty; exhaled nitric oxide; medication delivery devices; disease management; environmental remediation. [Respir Care 2015;60(5):744-748. (C) 2015 Daedalus Enterprises]
\end{abstract}

\section{Introduction}

Asthma is one of the most prevalent chronic respiratory diseases recognized in the United States, affecting $>25$ million people, including $>7$ million children. ${ }^{1}$ Common symptoms, including shortness of breath, wheezing, coughing, and chest tightness, are caused by inflammation, hyper-reactive airways, and bronchoconstriction and continue

Ms Deakins is affiliated with the Pediatric Respiratory Care Department, University Hospitals Rainbow Babies and Children's Hospital, Cleveland, Ohio.

Ms Deakins presented a version of this paper at the Year in Review 2014 of AARC Congress 2014, held December 9-12, 2014, in Las Vegas, Nevada.

Ms Deakins discloses relationships with Masimo and Hill-Rom.

Correspondence: Kathleen M Deakins MHA RRT-NPS FAARC, Women's and Children's Respiratory Care and Respiratory Diagnostic Services, to impact the lives of many. Asthma is a multifaceted disease with a need for growing evidence supporting key combinations of medications, techniques, and therapies to promote better health for all populations of patients.

In 2014, a PubMed search to identify updates on asthma yielded several hundred publications, which signifies a renewed focus on a need for better control and an ongoing pursuit for optimal treatment. This review on asthma was part of a series presented at the American Association for Respiratory Care Congress 2014, held in Las Vegas, Nevada, to address one of the topics of interest to the respiratory care community. In this paper, topics relevant to asthma are divided into 3 components: programs and guidelines, diagnosis and treatment, and management.

University Hospitals Rainbow Babies and Children's Hospital, 11100 Euclid Avenue, Cleveland, OH 44106. E-mail: kathleen.deakins@ uhhospitals.org.

DOI: $10.4187 /$ respcare. 04088 


\section{Programs and Guidelines}

In 2014, the National Heart, Lung, and Blood Institute recommended that the current guidelines established in 2012 become a living document with bi-annual updates instead of every 5-7 y. ${ }^{2}$ The National Heart, Lung, and Blood Advisory Council (a 37-member expert panel) met and reviewed the document "Expert panel report-3: guidelines for the diagnosis and management of asthma." Five priority topics were identified for literature review, and potential guideline updates were discussed. The role of medication dosing in wheezing and asthma, use of longacting antimuscarinic agents in asthma with inhaled corticosteroids, use of bronchial thermoplasty in adult severe exacerbations, role of the fraction of exhaled nitric oxide $\left(\mathrm{F}_{\mathrm{ENO}}\right)$ in diagnosis, medication selection and monitoring treatment response for asthma, and need for remediation of indoor allergens in asthma management were among the elements of proposed review and change.

The Global Initiative for Asthma updated its overall strategy of asthma management and prevention by redefining asthma to include variable limited expiratory flow as a finding, in addition to the already established classical symptoms of wheezing, coughing, and shortness of breath. ${ }^{2}$ In reference to diagnosis and management, approaches used to assess symptom control, identify risk factors, and proposed management strategies were reviewed. Asthma control was clearly defined by measuring the degree of limited activity, symptoms during sleep, daytime symptoms, need for rescue medication, lung function, and exacerbations. For patients using combination treatments such as inhaled corticosteroids and controller medications, regular follow-up was recommended at least every 6 months. To round out all aspects of care, recommendations involved reviewing asthma self-management practices, including asthma education, providing patient feedback on home treatment and monitoring, and arranging follow-ups. ${ }^{2}$

Other changes included altering the program related to management of pediatric patients below $5 \mathrm{y}$ of age. ${ }^{2}$ Relevant pediatric patient-specific information and recommendations were separated into their own distinct chapter. Patients with asthma and COPD (currently known as asthma-COPD overlap syndrome) were formally recognized to identify components of specific management related to this subset of asthma patients. The peer-reviewed documents with recommendations have become a resource for caregivers at every level.

Research programs typically provide important information on risk factors, management, and treatment of different populations of asthma patients. A well-known research program encompassing clinical studies involving both adult and pediatric asthma patients was merged into a single network. The National Heart, Lung and Blood Institute Asthma Network (AsthmaNet) devised a strategy to identify similarities and differences across age groups; to verify important interventions, data exchanges, and clinical outcomes; and to overcome operational challenges between populations of patients. ${ }^{3}$ Topics reviewed in the merged network include subject recruitment and specific consent challenges, demographics and populations, pediatric and adult asthma phenotypes, interventions related to control and outcomes, and growth and development. This combined network generated an enhanced repository of evidence-based information to be shared with clinicians participating at every level of care.

\section{Diagnosis and Treatment}

\section{Diagnosis}

Evidence supporting the relationship of risk factors and the incidence of asthma continues to unfold. Pediatric patients present unique challenges for diagnosis and management of asthma. Obesity has been linked to an increased incidence of asthma in children for a number of years. ${ }^{4}$ Unlike efforts to show further correlation between obesity and incidence of asthma using body mass index alone, Chen et $\mathrm{al}^{4}$ demonstrated a relationship between poor fitness level and an increase in sedentary time, in addition to the obesity factor, and a greater incidence of asthma in children. Based on $3 \mathrm{y}$ of these reproducible measurements, the findings showed that $\mathrm{FEV}_{1}$ values were decreased during pulmonary function tests in these pediatric subjects. Additional information about risk factors can be applied to individual patients for promoting early prevention and treatment interventions.

Classification by Phenotype. Phenotypes are used to identify factors that relate to asthma diagnosis. The identification of phenotypes in children has been a focus for asthma researchers. In 2014, Depner et al ${ }^{5}$ reviewed epidemiological factors of clinical phenotypes in children using a latent class analysis classification system. Clinical phenotypes used in these studies included subject history, components of a complete diagnostic evaluation, and treatment response. Subjects under 6 y of age with wheezing were monitored using: lung function, atopy, exhaled nitric oxide, and medications. The smallest subjects used the greatest number of resources to control wheezing. Children $\leq 5 \mathrm{y}$ are difficult to diagnose and treat, as there is little evidence that identifies consistency in successful control of this population. ${ }^{6}$ Attempts to identify phenotypes for preschool-age subjects have not been successfully validated; therefore, establishing treatment standards for this population has been a challenge. ${ }^{6}$

Ducharme et $\mathrm{al}^{6}$ acknowledged that additional risk factors such as environmental exposure, genetic susceptibility, and prenatal history predispose these patients to wheez- 
ing at a young age. Preschoolers presenting with wheezing and impaired lung function before starting first grade are likely to continue to have recurrent wheezing episodes throughout childhood and as young adults.

$\mathbf{F}_{\text {ENO }} \cdot \mathrm{F}_{\mathrm{ENO}}$ measurements are considered to be an index of airway inflammation measured as part of a diagnostic evaluation for asthma. The role $\mathrm{F}_{\mathrm{ENO}}$ plays in the diagnosis of asthma continues to evolve. In adults, $\mathrm{F}_{\mathrm{ENO}}$ typically decreases by $\sim 20 \%$ in response to bronchodilator administration. ${ }^{7}$ In 2014, further investigation into $\mathrm{F}_{\mathrm{ENO}}$ responses in pediatric patients was pursued. In a study conducted by Grzelewski et al, ${ }^{7}$ bronchodilator response at certain $\mathrm{F}_{\mathrm{ENO}}$ levels showed larger variances in pediatric subjects. Attempts to demonstrate alignment of $\mathrm{F}_{\mathrm{ENO}}$ with other asthma diagnostic indicators are forthcoming. Grzelewski et $\mathrm{al}^{7}$ evaluated the relationship between $\mathrm{FEV}_{1}$ and $\mathrm{F}_{\mathrm{ENO}}$ in pediatric asthma subjects. Although these relationships need further validation, the correlation between $\mathrm{FEV}_{1}$ and $\mathrm{F}_{\mathrm{ENO}}$ demonstrated that as $\mathrm{F}_{\mathrm{ENO}}$ increases, $\mathrm{FEV}_{1}$ decreases (up to $\mathrm{F}_{\mathrm{ENO}}$ of $35.4 \mathrm{ppb}$ ). The authors showed when $\mathrm{F}_{\mathrm{ENO}}$ was $>35.4 \mathrm{ppb}$, the pre-bronchodilator $\mathrm{FEV}_{1}$ increased. In subjects with moderate asthma, $\mathrm{FEV}_{1}$ and $\mathrm{F}_{\mathrm{ENO}}$ became more linear. To obtain diagnostically relevant information, measuring $\mathrm{F}_{\mathrm{ENO}}$ before and after a pre-bronchodilator and post-bronchodilator evaluation via spirometry in children was recommended. Using $\mathrm{F}_{\mathrm{ENO}}$ values as diagnostic parameters in pediatric patients is feasible through careful attention to physiologic differences and responses to medications in both adult and pediatric patient populations.

\section{Treatment}

Early detection and management of asthma symptoms are imperative to achieve successful treatment and control. In adults, corticosteroids and leukotriene receptor antagonists in combination with short- and long-acting $\beta_{2}$ agonists are the fundamentals of treatment. Inhaled corticosteroids are administered for the inflammatory component of asthma. Long-acting $\beta_{2}$ agonists and leukotriene antagonists are frequently part of the asthma symptom control regime for bronchial hyper-responsiveness. For patients who present with symptoms of wheezing while being treated with corticosteroids, a long-acting $\beta_{2}$ agonist is recommended. When added to an adult patient's treatment plan, long-acting $\beta_{2}$ agonists resulted in a reduction of exacerbations, improved $\mathrm{FEV}_{1}$, reduced symptoms, and improved quality of life. ${ }^{8}$ However, an increase in severe exacerbations was noted; therefore, a combination of long-acting $\beta_{2}$ agonists and inhaled corticosteroids in a single inhaler was recommended.

In pediatric patients, medications used to treat wheezing differ slightly. Bronchoconstriction in children with viral illness is often unaffected by administration of $\beta_{2}$ ago- nists. ${ }^{6}$ Intermittent corticosteroid therapy is recommended in situations in which moderate wheezing is caused by viral illness. ${ }^{6}$

Medication Delivery Devices. Aerosol delivery devices are evaluated and selected based on ease of use, treatment duration, medication delivery, and cost. Is a metered-dose inhaler used with a holding chamber equivalent to a nebulizer in terms of drug delivery, duration of time to complete technique, and stay in an acute care setting? In a Cochrane Review of 39 trials with 1,897 children and 797 adults, metered-dose inhalers with spacers were introduced in an emergency room setting to evaluate if they were as effective as nebulizer therapy. ${ }^{9}$ Pediatric subjects showed a significant reduction in adverse effects such as tachycardia, tremor, and emergency department stay, whereas adult subjects did not show a significant decrease in emergency department stay. Delivery of medications via nebulizer devices is not superior to metered-dose inhalers with spacers, and both methods are feasible.

Aerosol devices and patient technique impact medication delivery for asthma patients. Because many adult patient technique errors occur during inspiration (10-18\%), it is imperative that education and follow-up on basic concepts occur regularly. ${ }^{9,10}$ Fixed-dose medication combinations appear to promote a lower incidence of technique errors with inhalers. ${ }^{9}$ Better adherence was demonstrated when technique was reviewed, repeated, and followed up with training. ${ }^{10}$

Mechanical Ventilation and Asthma. For adults over $18 \mathrm{y}$ of age, asthma exacerbations account for $>2.4$ million hospital admissions in the United States. ${ }^{11}$ The number of patients admitted for asthma exacerbations is on the rise and accounts for a disturbing $60 \%$ of all admissions of patients $>50$ y of age. ${ }^{11}$ About $10 \%$ of these admissions require ICU care, with an average of $3 \%$ requiring mechanical ventilator support. ${ }^{11}$ In 2014, the Healthcare Cost and Utilization Project (National Inpatient Sample) evaluated subjects who were admitted with asthma exacerbations requiring invasive mechanical ventilation and noninvasive ventilation (NIV). Invasive mechanical ventilation and NIV rates combined increased by $45 \%$ over the $8-y$ evaluation period (2000-2008). ${ }^{11}$ The number of subjects requiring invasive mechanical ventilation decreased by $50 \%$, whereas those requiring NIV rose by $400 \%$ during the same time period. ${ }^{11}$ Mortality and hospital stay were unchanged despite the type of ventilation used. Although use of NIV during asthma exacerbations has increased significantly, the outcomes related to NIV practices need further investigation. The most critical asthma patients are at the highest risk, and data to support clinical practice associated with improved outcomes are forthcoming. 


\section{Novel Therapies}

Bronchial Thermoplasty. Bronchial thermoplasty, a nonpharmacologic therapy that applies radiofrequency energy in the form of heat to bronchial smooth muscle for ablation, is intended to reduce bronchospasm in cases of refractory (severe) asthma. ${ }^{12}$ Four y following its FDA clearance, bronchial thermoplasty has not been adopted as a standard, but is instead considered to be a novel therapy. Its clearance as a treatment for asthma was centered on prior studies, including the Asthma Intervention Research 2 trial, which compared bronchial thermoplasty-directed therapy with sham therapy and its effects on the Asthma Quality of Life Questionnaire. ${ }^{12}$ Although not part of the primary objective, secondary outcomes showed peak-flow changes, less need for rescue medications, and reductions in lost work days and severe asthma exacerbations. The outcomes for the sham and bronchial thermoplasty groups were not statistically different. It remains unclear whether one therapy is superior to the other. Although the criteria for bronchial thermoplasty remain stringent, including corticosteroid dependence, evidence of frequent exacerbations, in-patient admissions, history of emergency room visits, significant decrease in $\mathrm{FEV}_{1}$, and inadequate quality of life, there is a question as to whether the therapy needs additional investigation before its use. ${ }^{12}$

In search of direct evidence supporting clinical improvement from bronchial thermoplasty, Kaukel et $\mathrm{al}^{13}$ noted that these secondary outcomes revealed no significant change in $\mathrm{FEV}_{1}$ or airway hyper-responsiveness. A reduction in the number of severe exacerbations, as well as reductions in the number of missed work days and emergency room visits, was also noted. Because this intervention is not suitable for all severely affected asthma patients, identifying a phenotype that demonstrates response to bronchial thermoplasty is imperative.

\section{Management}

\section{Disease Management Programs}

Disease management programs are implemented to control asthma symptoms, improve quality of care, generate positive outcomes, and reduce cost. ${ }^{14}$ Through evidencebased guidelines, asthma education has been shown to promote self-management, including an understanding of medications, a review of drug-delivery techniques, and an organized plan that addresses different degrees of acute and chronic care and that empowers patients to control their disease.

Disease management programs have exhibited optimistic outcomes in decreasing hospitalizations, improving the number of patients who self-manage their disease, and helping patients set goals for improvement. A longitudinal population-based study carried out in Germany over a 4-y period demonstrated a 5-fold increase in participants and an increase in physician alignment with the program. ${ }^{14}$ Table 2 of this study displays the changes in the trends in prescribing medication over the 4-y period. Oral corticosteroid prescriptions decreased; inhaled corticosteroid use was not significantly changed; and long-acting $\beta_{2}$ agonist use and monotherapy increased. The most striking changes were a $50 \%$ increase in implementation of asthma home treatment plans and a 5-fold increase in self-management education. Collectively, these components of the program had a direct effect on reducing hospitalizations by $75 \%$. The use of disease management programs is gaining popularity. Systematic quality improvement displayed in the progress and outcomes of these programs has been demonstrated in nearly every aspect of each program. Sustainability in disease management may improve the future of asthma management.

\section{Environmental Remediation in Asthma Management}

The prevalence of an allergy-related environmental trigger component of asthma is on the rise. ${ }^{15}$ Environmental exposure to indoor allergens is believed to be a primary contributor to exacerbation, especially in children. A recent trend shows that the amount of time patients spend indoors contributes to increased exposure to household dust mites. ${ }^{15}$ Dust mites and mold are believed to be fostered in an environment of high humidity and temperature. Exposure to domestic pets, rodents, cockroaches, tobacco smoke, and air pollution may create allergy-related triggers that produce symptoms. The latest updates on environmental remediation collected from a number of studies were presented in $2014 . .^{15}$

The goal of sensitizing patients to allergens using a single intervention has not been shown to be successful in reducing reactions to allergens such as dust mites..$^{15}$ Fiftyfour studies conducted to assess dust mite interventions revealed that compared with control groups, there was no significant change in asthma symptoms despite interventions. ${ }^{15}$ Small changes in 1-y corticosteroid use in a single study could not be reproduced in similar evaluations. Documented interventions used to reduce dust mite exposure include routine cleaning; washing bedding weekly; and eliminating carpets, stuffed animals, and some furniture. A multifaceted approach to remediation is recommended.

Pet-related exposures causing asthma-related symptoms are most easily remediated by removal of a pet from the home. Corticosteroid use was not necessary when the pet was removed, but was high when the pet remained in the house. ${ }^{15}$ When pets were allowed to remain in the home, the use of high-efficiency particulate filters was not shown to be a sustained remediation measure. 
Cockroach and rodent exposure in densely populated areas was found in as many as half of all homes studies. ${ }^{15}$ The National Cooperative Inner-City Asthma Study showed that allergen exposure could be reduced through a combination of pest control initiatives and patient education. ${ }^{15}$ Remediation of infestation is challenging and requires a combination of methods to be successful.

Mold and endotoxin exposure is prevalent in inner city areas, where a damp environment, inadequate ventilation, and poor air quality exist. Awareness of endotoxin exposure has become more widespread following Hurricane Katrina. ${ }^{15}$ Cleaning and, in some cases, disposal of damaged house furnishings were required to reduce symptoms. Allergens from mold exposure have been associated with an increased number of asthma-related symptoms. Aggressive interventions for mold eradication resulted in reduced emergency room visits and hospitalizations for 62 asthma subjects compared with a control group. ${ }^{15}$

Anecdotally, going back to school is believed to result in an increased incidence of asthma exacerbations. Endotoxin levels in dust in schools were higher than those at home, indicating that this viewpoint may indeed be true, at least in inner city schools. ${ }^{15}$

Other endotoxins such as nicotine and nitrogen dioxide found in air pollution have adverse effects on patients with asthma and can produce troublesome symptoms. ${ }^{15}$ Nitrogen dioxide exposure in young children has been associated with coughing and wheezing in the first few years of life.

Environmental smoke exposure is a known irritant and cause of asthma-related symptoms. However, despite interventions to reduce exposure, there is no proven effective method to eliminate its side effects. ${ }^{15}$ Although avoidance has been shown to be the most effective measure, high-efficiency particulate air filter use may provide some benefit in reducing acute symptoms from smoke exposure of any kind. Environmental exposure continues to play an important role in triggering asthma symptoms. Continued emphasis on preventing exposure and remediation strategies are integral to maintaining asthma control.

\section{Summary}

In this review, important recent literature on key topics related to asthma were presented. Diagnosis, treatment, and management were reviewed. Key concepts from updates available in 2014 were presented.

\section{REFERENCES}

1. Moorman JE, Akinbami LJ, Bailey CM, Zahran HS, King ME, Johnson CA, Liu X. National surveillance of asthma: United States, 20012010. Vital Health Stat 3 2012;(35):1-67.

2. National Heart, Lung, and Blood Advisory Council Asthma Expert Working Group. Needs assessment for potential update of expert panel report-3 (2007): guidelines for the diagnosis and management of asthma April 2014. https://www.nhlbi.nih.gov/sites/ www.nhlbi.nih.gov/files/NHLBAC-Asthma-WG-Report.pdf. Accessed October 3, 2014.

3. Cabana MD, Kunselman SJ, Nyenhuis SM, Wechsler ME. Researching asthma across the ages: insights from the National Heart, Lung and Blood Institute's Asthma Network. J Allergy Clin Immunol 2014;133(1):27-33.

4. Chen YC, Tu YK, Huang KC, Chen PC, Chu DC, Lee YL. Pathway from central obesity to childhood asthma: physical fitness and sedentary time are leading factors. Am J Respir Crit Care Med 2014; 189(10):1194-1203.

5. Depner M, Fuchs O, Genuneit J, Karvonen AM, Hyvärinen A, Kaulek $\mathrm{V}$, et al. Clinical and epidemiologic phenotypes for childhood asthma. Am J Respir Crit Care Med 2014;189(2):129-138.

6. Ducharme FM, Tse SM, Chauhan B. Diagnosis, management and prognosis of preschool wheeze. Lancet 2014;383(9928):1593-1604.

7. Grzelewski T, Majak P, Jerzyńska J, Stelmach W, Stelmach R, Janas $\mathrm{A}$, et al. Interpretation of exhaled nitric oxide values in children with asthma depends on degree of bronchoconstriction and the levels of asthma severity. Respir Care 2014;59(9):1404-1411.

8. Chauhan BF, Ducharme FM. Addition to inhaled corticosteroids of long acting beta ${ }_{2}$-agonists versus anti-leukotrienes for chronic asthma. Cochrane Database Syst Rev 2014;1:CD003137.

9. Cates CJ, Welsh EJ, Rowe BH. Holding chambers (spacers) versus nebulisers for beta-agonist treatment of acute asthma. Cochrane Database Syst Rev 2013;9:CD000052.

10. Yildiz F, Asthma Inhaler Treatment Study Group. Importance of inhaler device use status in the control of asthma in adults: the asthma inhaler treatment study. Respir Care 2014;59(2):223-230.

11. Nanchal R, Kumar G, Majumdar T, Taneja A, Patel J, Dagar G, et al. Utilization of mechanical ventilation for asthma exacerbations: analysis of a national database. Respir Care 2014;59(5):644-653.

12. Iyer VN, Lim KG. Bronchial thermoplasty: reappraising the evidence (or lack thereof). Chest 2014;146(1):17-21.

13. Kaukel P, Herth FJ, Schuhmann M. Bronchial thermoplasty: interventional therapy in asthma. Ther Adv Respir Dis 2014;8(1):22-29.

14. Mehring M, Donnachie E, Mutschler R, Hofmann F, Keller M, Schneider A. Disease management programs for patients with asthma in Germany: a longitudinal population-based study. Respir Care 2013; 58(7):1170-1177.

15. Wright LS, Phipatanakul W. Environmental remediation in the treatment of allergy and asthma: latest updates. Curr Allergy Asthma Rep 2014;14(3):419. 